\title{
Candida auris: Emergência Recente de um Fungo Patogénico Multirresistente
}

\section{Candida auris: The Recent Emergence of a Multiresistant Pathogenic Fungi}

\author{
Francisco ANTUNES $₫ 1$, Cristina VERÍSSIMO ${ }^{2}$, Álvaro Ayres PEREIRA ${ }^{1,3}$, Raquel SABINO ${ }^{1,2}$ \\ Acta Med Port 2020 Oct;33(10):680-684 - https://doi.org/10.20344/amp.12419
}

\begin{abstract}
RESUMO
Candida auris foi descrita pela primeira vez em 2009, como uma espécie distinta das outras leveduras do género Candida. Este fungo é difícil de se distinguir de outras espécies com que se correlaciona, sendo, atualmente, aconselhada a confirmação da sua identificação por laboratórios de referência. Esta levedura tem a capacidade de causar infeções invasivas, podendo ser resistente às três classes de antifúngicos, sendo a mortalidade elevada. Candida auris tem sido detetada em vários países do Mundo, incluindo alguns países da Europa, tais como Reino Unido e Espanha. Até à data, em Portugal, não há registo de qualquer caso de infeção/colonização por Candida auris. Esta espécie pode sobreviver no ambiente durante várias semanas e uma vez introduzida no ambiente hospitalar, o risco de transmissão é elevado, obrigando à implementação de medidas rigorosas de controlo da infeção, por forma a prevenir a transmissão. Este artigo pretende dar a conhecer e discutir a problemática da emergência deste fungo e as suas eventuais consequências. Palavras-chave: Candida; Farmacorresistência Fúngica Múltipla; Infecção Hospitalar
\end{abstract}

\section{ABSTRACT}

Candida auris was first described as a new species back in 2009. Although it differs markedly from other Candida species, this species can be misidentified as other yeasts in the routine microbiology laboratories. Therefore, its identification to species level should be confirmed by reference laboratories. Candida auris exhibits potential to cause invasive infections, and frequently shows a multidrugresistance pattern, and it is associated with high mortality rates. Outbreaks caused by Candida auris and associated with health care institutions have been reported in several countries around the world, including some European countries, such as the United Kingdom and Spain. In Portugal, to our knowledge, there are no known infections or colonization cases caused by Candida auris. This species can survive in the environment for several weeks and once introduced into the hospital environment, the risk of transmission is high, requiring strict infection control measures in order to prevent transmission. This paper intends to raise the awareness of the emergence of this fungal species, as well as to discuss the consequences of this situation.

Keywords: Candida; Cross Infection; Drug Resistance, Multiple, Fungal

\section{INTRODUÇÃO}

A emergência mundial de Candida auris (C. auris), tem sido referida como uma ameaça à saúde pública, dada a elevada percentagem de isolados resistentes a uma ou mais classes de antifúngicos disponíveis. Esta espécie, descrita pela primeira vez em 2009, tem o potencial de causar infeções invasivas que podem disseminar a nível inter- e intra-hospitalar e às quais está associada elevada mortalidade. ${ }^{1}$ No entanto, as taxas de mortalidade variam significativamente entre diferentes regiões geográficas e unidades hospitalares. ${ }^{2} \mathrm{~A}$ maioria dos fatores de risco relacionados com infeção por $C$. auris não são diferentes dos associados a infeções causadas por outras espécies de Candida. ${ }^{3-6}$ Como tal, a elevada mortalidade atribuível a $C$. auris não pode ser estabelecida facilmente. O número de mortes atribuíveis a infeções por $C$. auris, em oposição às condições médicas subjacentes, pode ser difícil de quantificar. $^{2,7}$ Esta nova espécie de Candida, potencialmente patogénica, apresenta, para além da multirresistência, a capacidade de persistência em meio hospitalar (superfícies, dispositivos e equipamentos médicos), sendo difícil a sua eliminação. Por outro lado, C. auris tem sido isolada a partir da colonização da pele, nomeadamente das axilas e das virilhas, bem como de outras regiões do corpo, nas quais pode persistir por períodos longos. A dificuldade na identificação laboratorial pelos métodos convencionais, a capacidade de produzir bio filmes, o potencial para transmissão horizontal no ambiente hospitalar, os seus mecanismos de virulência, a predisposição para escapar aos mecanismos habituais de defesa imunitária do hospedeiro, principalmente da ação dos neutrófilos e, ainda, a eficácia subóptima dos produtos usados na desinfeção ambiental nos hospitais são características desta espécie emergente. ${ }^{8-12} \mathrm{~A}$ sua virulência é comparável à de Candida albicans, considerada a espécie mais patogénica do género. ${ }^{13}$ Para além de outras características (germinação, aderência, formação de bio filme, atividade da hemolisina, produção de fosfolipases e de proteinases), a sua termo-tolerância (> $42^{\circ} \mathrm{C}$ ), e a sua tolerância a concentrações salinas elevadas, podem potenciar a sua sobrevivência e patogenicidade. ${ }^{8}$

\section{METODOLOGIA}

Procedeu-se a uma revisão bibliográfica dos artigos mais relevantes sobre $C$. auris. Para tal, como metodologia adoptada, efectuou-se busca nos motores de pesquisa

1. Instituto de Saúde Ambiental. Faculdade de Medicina. Universidade de Lisboa. Lisboa. Portugal.

2. Departamento de Doenças Infeciosas. Unidade de Referência de Infeções Parasitárias e Fúngicas. Instituto Nacional de Saúde Dr. Ricardo Jorge. Lisboa. Portugal.

3. Serviço de Doenças Infecciosas. Hospital de Santa Maria. Centro Hospitalar Universitário Lisboa Norte. Lisboa. Portugal.

$\triangle$ Autor correspondente: Francisco Antunes. fantunes@medicina.ulisboa.pt

Recebido: 06 de junho de 2019 - Aceite: 17 de outubro de 2019 | Copyright @ Ordem dos Médicos 2020 
Google Scholar e PubMed utilizando as palavras-chave "Candida auris", "Candida epidemiology", "antifungal resistance", "Candida therapy", "Candida auris infection control", "Candida auris outbreaks" e "Candida auris risk factors".

\section{RESULTADOS}

\section{Características microbiológicas de C. auris}

Os diferentes sistemas bioquímicos, disponíveis no mercado para a identificação de fungos levedurifomes, podem não distinguir $C$. auris das espécies com que se correlaciona, pelo que esta tem sido confundida com outros microrganismos, tais como $C$. haemulonii e Saccharomyces cerevisiae. Alguns laboratórios não reconhecem Candida ao nível da espécie, pelo que $C$. auris tem sido referida como "outras Candida spp.". A identificação correta de $C$. auris poderá ser realizada através de métodos bioquímicos ou de espectroscopia de massa, desde que os equipamentos possuam as bases de dados atualizadas, ou seja, que integrem já o perfil desta espécie.

As suas características fenotípicas e genotípicas sugerem que se trata de uma nova espécie do género Candida, da classe Ascomycetes. O desenvolvimento de testes de reação em cadeia de polimerase (PCR - do inglês polymerase chain reaction) para $C$. auris tem-se revelado promissor para a identificação rápida e precisa deste fungo, em particular no contexto de surtos. ${ }^{14,15}$ Assim, o European Centre for Disease Prevention and Control (ECDC) recomenda que isolados provenientes de hemoculturas e de amostras colhidas de locais habitualmente estéreis, identificados como $C$. auris ou com suspeita de pertencer a esta espécie, sejam encaminhados para laboratórios de referência para caracterização molecular. ${ }^{16}$ Também as leveduras, com identificação realizada em sistemas sem base de dados atualizada e que tenham perfil compatível com Candida famata, Candida sake, Candida haemulonii (complexo), Saccharomyces cerevisiae e Rhodotorula glutinis (sem pigmentação rosa/laranja), bem como leveduras do género Candida, cuja espécie não seja identificada pelos métodos bioquímicos convencionais, devem seguir a mesma recomendação.

\section{Epidemiologia}

Levando em linha de conta que este fungo patogénico tem a capacidade de sobrevivência e de persistência no meio ambiente clínico, com aptidão para a colonização rápida da pele dos doentes e de transmissibilidade elevada, é de esperar o aumento da sua frequência a nível global, com surtos graves e prolongados. A colonização no doente pode persistir várias semanas após o contágio inicial e, da mesma forma, o período temporal de sobrevivência desta espécie em superfícies é, também, longo. ${ }^{2}$

A emergência global desta espécie, em regiões geograficamente restritas, tem sido registada. De acordo com estudos recentes, usando a metodologia de sequenciação total do genoma (WGS - do inglês Whole Genome Sequencing), a população mundial de $C$. auris consiste em quatro clades distintas (Ásia Oriental, Ásia Meridional,
África e América do Sul), o que corrobora a hipótese de transmissão clonal. ${ }^{1}$ A prevalência e as características epidemiológicas de $C$. auris estão, ainda, por clarificar, sendo uma das causas a subestimação do seu isolamento, dada a limitação na sensibilidade das técnicas disponíveis, na prática corrente dos laboratórios de microbiologia clínica ${ }^{17}$. Antes de 1996, não há notícia de identificação de C. auris, previamente reconhecida como tal, e até 2008 são conhecidos, apenas, quatro isolados..$^{1,18} \mathrm{Em} \mathrm{2009}$, esta espécie foi descrita, pela primeira vez, numa amostra de exsudado do ouvido externo. ${ }^{8}$ Posteriormente, foram identificados 15 doentes, com otite média crónica causada por C. auris. ${ }^{19}$ Mais tarde, ficou estabelecido que esta espécie nova tinha a capacidade de causar infeções invasivas. ${ }^{18}$

A partir destes isolamentos, C. auris foi assinalada em múltiplas regiões geográficas, em particular na Índia, onde a sua prevalência, em casos de candidemia, atinge $17,5 \%{ }^{20}$ Quer nos Estados Unidos da América (EUA), quer na Europa têm sido referidos, cada vez com maior frequência, casos de colonização e de infeção por $C$. auris, alguns deles importados, com subsequente transmissão local e com surtos nosocomiais. ${ }^{18,21-23} \mathrm{Na}$ Europa, o primeiro surto ocorreu no Reino Unido, num Hospital de Londres, de 2015 a 2016 (16 meses), com 50 casos de C. auris, sendo a maioria de colonização da pele, com $44 \%$ de infeção e $18 \%$ de candidemia, entre os doentes colonizados. ${ }^{24}$ Seguidamente, em Espanha, surgiu um outro surto, descrito entre abril de 2016 e janeiro de 2017. Durante este período, foram detetados 140 doentes colonizados (68\% deles internados em unidades de cuidados intensivos) e 41 com infeção da corrente sanguínea ( $87,8 \%$ da unidade de cuidados intensivos cirúrgicos). ${ }^{23,26} \mathrm{Em}$ Portugal, não há registo, até à presente data, de nenhum caso de infeção/colonização por $C$. auris, não existindo, também, registo de isolamentos desta espécie no ambiente. Em maio de 2019, o Instituto Nacional de Saúde Doutor Ricardo Jorge (INSA), em colaboração com o Instituto de Saúde Ambiental (ISAMB) e com o Centro Hospitalar Universitário Lisboa Norte (CHULN) deu início a um estudo exploratório, para deteção de $C$. auris em amostras ambientais e nas mãos dos prestadores de cuidados de saúde de uma unidade deste Centro Hospitalar. Este estudo, o primeiro deste género efetuado em Portugal, contribuirá com informação necessária para a prevenção de eventuais surtos causados por este fungo e para um meIhor conhecimento da sua epidemiologia.

\section{Características clínicas e factores de risco}

As características clínicas da infeção por $C$. auris são similares às causadas por outras espécies de Candida. Os isolados de $C$. auris têm sido obtidos de diferentes locais do organismo, para além daqueles reportados a partir de dispositivos invasivos e das pontas dos cateteres. Os isolados obtidos de regiões não estéreis do organismo (orofaringe, pulmão, trato urinário e aparelho genital [vulvo-vaginite], pele e tecidos moles) representam mais colonizações do que infecções. ${ }^{24}$ No entanto, a colonização implica risco de transmissão, que requer a implementação de medidas de 
controlo da infeção.

As infeções invasivas são definidas quando os exemplares são obtidos de locais, em condições normais estéreis (sangue, líquidos cefalorraquidiano, pleural e articular). ${ }^{27}$ Os isolados do corrimento do canal auditivo externo correspondem, principalmente, a casos de colonização (por inoculação direta por equipamento médico contaminado), sendo as infeções invasivas pouco comuns. ${ }^{28}$ Numa unidade de cuidados intensivos de neurocirurgia, dos 70 doentes colonizados por $C$. auris, apenas sete desenvolveram infeções invasivas, estando o surto epidémico associado à utilização de termómetros axilares reutilizáveis. ${ }^{23}$ Num estudo na Índia, em unidades de cuidados intensivos, foram identificados 1400 casos de candidemia, dos quais $5,3 \%$ atribuído a $C$. auris. ${ }^{4} \mathrm{~A}$ duração do internamento foi mais longa, a exposição a antifúngicos foi maior, a duração da presença de cateteres centrais foi mais prolongada e a alimentação parentérica significativamente mais frequente, comparando os casos de candidemia por $C$. auris com não-auris. Quanto aos fatores de risco não foram encontradas diferenças entre as infeções invasivas por $C$. auris e as outras espécies de Candida, tais como a prévia exposição a antibióticos e a antifúngicos, diabetes mellitus, cirurgia abdominal e vascular, presença de cateteres venosos centrais, cateterização urinária, drenos pós-operatórios, doença renal crónica, alimentação parentérica total, terapêutica imunossupressora e neutropenia e, ainda, duração de internamento em unidade de cuidados intensivos.

Em regra, a infeção ocorre algumas semanas depois da hospitalização e a mortalidade varia de $30 \%$ a $72 \%$, refletindo, possivelmente, maior patogenicidade do que a de outras espécies de Candida. ${ }^{1,5,11,25,29} \mathrm{~A}$ variação na mortalidade é devida, principalmente, às condições subjacentes destes doentes, o que confunde a mortalidade atribuída a C. auris. ${ }^{30}$

\section{Resistência aos antifúngicos}

Os isolados de $C$. auris têm mostrado multirresistência aos antifúngicos, com uma frequência muito superior à das outras espécies de Candida. ${ }^{31}$ Tal como algumas espécies de Candida, C. auris tem a capacidade de formar bio filmes, que conferem maior resistência aos antifúngicos de todas as classes (azóis, polienos e equinocandinas) e, por outro lado, a resistência a estas três classes de antifúngicos é caracterizada por diversos mecanismos, como mutações no gene $E R G 11$, responsável pela resistência aos azóis e nos genes associados à atividade da bomba de efluxo. ${ }^{1,32,33}$

Se bem que não estejam estabelecidos os valores limites de concentrações mínimas inibitórias (CMls) para $C$. auris, de um modo geral, quase todos os isolados apresentam resistência in vitro ao fluconazol, mais de metade ao voriconazol, um terço à anfotericina $B$ e alguns às equinocandinas. ${ }^{34}$ Num elevado número de isolados, as CMls para o fluconazol são elevadas (> $64 \mathrm{mg} / \mathrm{L}$ ); este padrão de resistência é comum a isolados provenientes de diferentes zonas geográficas. No entanto, a resistência ao fluconazol não é intrínseca desta espécie. Por outro lado, foram também reportados casos nos Estados Unidos em que ocorreu insucesso do tratamento com fluconazol, em isolados sensíveis a este antifúngico. ${ }^{35}$

Num estudo efetuado em 350 isolados de C. auris provenientes da Índia 90\% destes apresentou resistência ao fluconazol, $2 \%$ à anidulafungina, $2 \%$ à micafungina e $8 \%$ à anfotericina B. ${ }^{22,33}$ Nos Estados Unidos, cerca de $90 \%$ dos isolados foram resistentes ao fluconazol, $30 \%$ à anfotericina $B$ e $5 \%$ às equinocandinas. ${ }^{34}$ Dos isolados testados no Reino Unido, $90 \%$ apresentou resistência ao fluconazol, aproximadamente $20 \%$ à anfotericina B e cerca de $10 \%$ às equinocandinas. $^{2}$

Alguns isolados mostraram CMls elevadas para as três classes de antifúngicos, limitando, assim, as opções terapêuticas. No modelo animal, a micafungina foi, das três classes de antifúngicos, a que apresentou maior atividade antifúngica. ${ }^{36}$

\section{Tratamento}

O tratamento da candidemia inclui, não apenas a prescrição de antifúngicos, mas, também, uma série de outras medidas, tais como a drenagem de abcessos, a remoção dos cateteres venosos centrais e de outros dispositivos médicos invasivos, a monitorização da eficácia da terapêutica por culturas de sangue e, no caso destas persistirem positivas, a exclusão dos potenciais focos metastásicos, como abcessos e a endocardite. ${ }^{37,38}$

Todos os episódios iniciais de candidemia por C. auris devem ser tratados com equinocandinas ${ }^{27}$ Em caso de candidemia persistente ( $>$ cinco dias, após o início da terapêutica), a anfotericina $B$ lipossómica ou o voriconazol, podem ser combinados com a equinocandina. A duração da terapêutica antifúngica, na candidemia, é determinada pela resposta clínica e micológica e, em princípio, deve prolongar-se por, pelo menos, mais duas semanas após a negativação das culturas. ${ }^{39}$

\section{Prevenção e controlo}

Uma vez introduzida no ambiente, $C$. auris acarreta risco de transmissão e é difícil e dispendiosa a sua erradicação. Todos aqueles doentes colonizados ou com infeções por $C$. auris, além das precauções básicas de controlo de infeção devem ser submetidos a medidas de precaução de contacto. ${ }^{16}$

De acordo com o $\mathrm{CDC}^{40}$ (Centers for Disease Control and Prevention), todos os doentes colonizados ou infetados deverão estar num quarto individual ou num quarto com outros doentes colonizados por $C$. auris; caso o doente esteja colonizado com $C$. auris e outros microrganismos multirresistentes deverá ser colocado com doentes colonizados com os mesmos microrganismos multirresistentes. Não se recomenda a partilha de quarto de doentes apenas colonizados por $C$. auris com outros doentes colonizados com outros microrganismos. As precauções de prevenção e controlo de infeção deverão ser reforçadas, com especial atenção à higienização das mãos e ao uso de equipamento de proteção individual descartável, de uma única 
utilização. ${ }^{41} \mathrm{O}$ equipamento partilhado por diferentes doentes deverá ser limpo e desinfetado após cada utilização. ${ }^{40}$

Dado que $C$. auris pode persistir no ambiente várias semanas, a presença de um doente com colonização ou infeção por $C$. auris obriga a um reforço da descontaminação das superfícies de toque frequente e à desinfeção terminal da respetiva unidade, quando da alta do doente. Esta desinfeção poder-se-á fazer com desinfetantes à base de cloro com uma potência de 1000 ppm (superior à usada rotineiramente) ou, quando da desinfeção terminal, utilizando técnicas com vapor de peróxido de hidrogénio ou com radiação ultravioleta. ${ }^{16,42}$ Ao transferir os doentes para outras unidades de saúde, estas deverão ser notificadas sobre o status de infeção ou colonização do doente e o nível de precauções recomendadas. ${ }^{40}$ Todos os doentes que tiveram contacto direto com um doente colonizado/infetado por $C$. auris devem ser rastreados para a presença deste fungo, em especial, nas amostras das axilas e das virilhas e se se detetar a sua presença deverão ser submetidos a medidas de precaução de contacto e ser ponderada a respetiva descolonização, embora não seja unanime o método ou antisséptico a utilizar. ${ }^{9,23,42,43}$

O controlo, em meio hospitalar, de surtos epidémicos causados por $C$. auris é um desafio difícil de superar, mas a redução do risco de transmissão deve-se fundamentar essencialmente, também, nas estratégias utilizadas, para os outros microrganismos, incluindo a utilização de equipamento de proteção individual e a rigorosa higienização das mãos. ${ }^{44}$ De acordo com as recomendações das instituições Public Health England e Centre for Opportunistic, Tropical and Hospital Infections, após a lavagem com sabão, as mãos deverão ser desinfetadas com uma solução à base de álcool, antes e depois de tratar do doente. ${ }^{41,42}$

\section{CONCLUSÃO}

A identificação correta da espécie C. auris e a determi- nação da sua suscetibilidade aos antifúngicos é essencial para o controlo da colonização ou da infeção do doente e prevenção da disseminação deste agente. Várias questões permanecem ainda por esclarecer, nomeadamente a prevalência de $C$. auris na população, origem, nicho ambiental, eficácia da prevenção, controlo de infeção e impacto na mortalidade dos doentes. ${ }^{7}$ É essencial um maior conhecimento sobre como ocorre a sua disseminação e quais são os possíveis reservatórios. Como tal, reveste-se de particular importância conhecer a sua ecologia, ambiente natural e a distribuição desta espécie. Também é importante explorar possíveis razões para esta recente emergência, como a presença ambiental de antifúngicos ou o efeito das mudanças climáticas. ${ }^{45}$

Estudos sobre a sua prevalência na comunidade permitirão esclarecer se $C$. auris está associada principalmente a infeções hospitalares ou se há disseminação entre ambiente hospitalar e a comunidade. ${ }^{7}$

Em relação ao próprio microrganismo, estudos em sequenciação do genoma completo são necessários para revelar mecanismos de resistência e fatores de virulência de forma a poderem, posteriormente, ser usados como novos alvos terapêuticos. Para além disso, mais dados sobre o padrão de suscetibilidade desta espécie aos diferentes antifúngicos permitirão estabelecer valores de corte epidemiológico desta espécie.

Apesar do que já foi descoberto até à atualidade, as comunidades científica e clínica necessitam de mais investigação e estudos seriados, de forma a desenvolver estas temáticas.

\section{CONFLITOS DE INTERESSE}

Os autores declaram não ter conflitos de interesse.

\section{FONTES DE FINANCIAMENTO}

Não foi solicitada qualquer fonte de financiamento.

\section{REFERÊNCIAS}

1. Lockhart SR, Etienne KA, Vallabhaneni S, Farooqi J, Chowdhary A, Govender NP, et al. Simultaneous emergence of multidrug-resistant Candida auris on 3 continents confirmed by whole-genome sequencing and epidemiological analyses. Clin Infect Dis. 2017;64:134-40.

2. Forsberg K, Woodworth K, Walters M, Berkow EL, Jackson B, Chiller $\mathrm{T}$, et al. Candida auris: the recent emergence of a multidrug-resistant fungal pathogen. Med Mycol. 2019;1;57:1-12.

3. Park JY, Bradley N, Brooks S, Burney S, Wassner C. Management of patients with Candida auris fungemia at community hospital, Brooklyn, New York, USA, 2016-2018. Emerg Infect Dis. 2019;25:601-2.

4. Rudramurthy SM, Chakrabarti A, Paul RA, Sood P, Kaur H, Capoor MR, et al. Candida auris candidaemia in Indian ICUs: analysis of risk factors. J Antimicrob Chemother. 2017;72:1794-801.

5. Osei SJ. Candida auris: a systematic review and meta-analysis of current updates on an emerging multidrug-resistant pathogen. MicrobiologyOpen. 2018;7:e00578.

6. Britz E, Govender NP. Global emergence of a multi-drug resistant fungal pathogen, Candida auris. Southern Africa J Infect Dis. 2016;31:69-70.

7. Jeffery-Smith A, Taori SK, Schelenz S, Jeffery K, Johnson EM, Borman A, et al; Candida auris Incident Management Team. Candida auris: a review of the literature. Clin Microbiol Rev. 2017;31.pii: e00029-17.

8. Satoh K, Makimura K, Hasumi Y, Nishiyama Y, Uchida K, Yamaguchi $\mathrm{H}$. Candida auris sp. nov., a novel ascomycetous yeast isolated from the external ear canal of an inpatient in a Japanese hospital. Microbiol

Immunol. 2009;53:41-4.

9. Chowdhary A, Sharma C, Meis JF. Candida auris: a rapidly emerging cause of hospital-acquired multidrug-resistant fungal infections globally. PLoS Pathog. 2017;13:e1006290.

10. Sherry L, Ramage G, Kean R, Borman A, Johnson EM, Richardson MD, et al. Biofilm-forming capability of highly virulent, multidrug-resistant Candida auris. Emerg Infect Dis. 2017;23:328-31.

11. Ku TS, Walraven CJ, Lee SA. Candida auris: disinfectants and implications for infection control. Front Microbiol. 2018;9:726.

12. Johnson CJ, Davis JM, Huttenlocher A, Kernien JF, Nett JE. Emerging fungal pathogen Candida auris evades neutrophil attack. MBio. 2018;9. pii: e01403-18

13. Borman AM, Szekely A, Johnson EM. Comparative pathogenicity of United Kingdom isolates of the emerging pathogen Candida auris and other key pathogenic Candida species. mSphere. 2016;1.pii:e00189-16.

14. Kordalewska M, Zhao Y, Lockhart SR, Chowdhary A, Berrio I, Perlin DS Rapid and accurate molecular identification of the emerging multidrugresistant pathogen Candida auris. J Clin Microbiol. 2017;55:2445-52.

15. Leach L, Zhu Y, Chaturvedi S. Development and validation of a realtime PCR assay for rapid detection of Candida auris from surveillance samples. J Clin Microbiol. 2018;56:e01223-17.

16. European Centre for Disease Prevention and Control. Candida auris in healthcare settings - Europe - first update, 23 April 2018. Stockholm: ECDC; 2018. 
17. Lockhart SR, Berkow EL, Chow N, Welsh RM. Candida auris for the clinical microbiology laboratory: not your grandfather's Candida species. Clin Microbiol Newsl. 2017;39:99-103.

18. Lee WG, Shin JH, Uh Y, Kang MG, Kim SH, Park KH, et al. First three reported cases of nosocomial fungemia caused by Candida auris. $\mathrm{J}$ Clin Microbiol. 2011;49:3139-42.

19. Oh BJ, Shin JH, Kim MN, Sung H, Lee K, Joo MY, et al. Biofilm formation and genotyping of Candida haemulonii, Candida pseudohaemulonii, and a proposed new species (Candida auris) isolates from Korea. Med Mycol. 2011;49:98-102

20. LeMathur P, Hasan F, Singh PK, Malhotra R, Walia K, Chowdhary A. Five-year profile of candidaemia at an Indian trauma centre: high rates of Candida auris blood stream infections. Mycoses. 2018;61:674-80.

21. Chow NA, Gade L, Tsay SV, Forsberg K, Greenko JA, Southwick KL, et al. US Candida auris Investigation Team. Multiple introductions and subsequent transmission of multidrug-resistant Candida auris in the USA: a molecular epidemiological survey. Lancet Infect Dis. 2018;18:1377-84.

22. Rhodes J, Abdolrasouli A, Farrer RA, Cuomo CA, Aanensen DM, Armstrong-James D, et al. Genomic epidemiology of the UK outbreak of the emerging human fungal pathogen Candida auris. Emerg Microbes Infect. 2018;7:43

23. Eyre DW, Sheppard AE, Madder H, Moir I, Moroney R, Quan TP, et al. A Candida auris outbreak and its control in an intensive care setting. N Engl J Med. 2018;379:1322-31.

24. Schelenz S, Hagen F, Rhodes JL, Abdolrasouli A, Chowdhary A, Hall A, et al. First hospital outbreak of the globally emerging Candida auris in a European hospital. Antimicrob Resist Infect Control. 2016;5:35.

25. Ruiz Gaitán AC, Moret A, López Hontangas JL, Molina JM, Aleixandre López Al, Cabezas $\mathrm{AH}$, et al. Nosocomial fungemia by Candida auris: first four reported cases in continental Europe. Rev Iberoam Micol. 2017;34:23-7.

26. Ruiz-Gaitán A, Moret AM, Tasias-Pitarch M, Aleixandre-López Al, Martínez-Morel H, Calabuig E, et al. An outbreak due to Candida auris with prolonged colonisation and candidaemia in a tertiary care European hospital Mycoses. 2018;61:498-505.

27. Center for Disease Control and Prevention. Candida auris 2018. Case definition [homepage na Internet]; [consultado 2019 mai 2]. Disponível em https://wwwn.cdc.gov/nndss/conditions/candida-auris/casedefinition/2018/.

28. Jung J, Kim MJ, Kim JY, Lee JY, Kwak SH, Hong MJ, et al. Candida auris colonization or infection of the ear: A single-center study in South Korea from 2016 to 2018. Med Mycol. 2019.pii: myz020. [Epub ahead of print].

29. Chakrabarti A, Sood P, Rudramurthy SM, Chen S, Kaur H, Capoor M, et al. Incidence, characteristics and outcome of ICU-acquired candidemia in India. Intensive Care Med. 2015;41:285-95

30. Chowdhary A, Sharma C, Duggal S, Agarwal K, Prakash A, Singh PK et al. New clonal strain of Candida auris, Delhi, India. Emerg Infect Dis. 2013;19:1670-3.

31. Sarma S, Upadhyay S. Current perspective on emergence, diagnosis and drug resistance in Candida auris. Infect Drug Resist. 2017;10:15565.

32. Chatterjee S, Alampalli SV, Nageshan RK, Chettiar ST, Joshi S, Tatu US. Draft genome of a commonly misdiagnosed multidrug resistant pathogen Candida auris. BMC Genomics. 2015;16:686.

33. Chowdhary A, Prakash A, Sharma C, Kordalewska M, Kumar A, Sarma $\mathrm{S}$, et al. A multicentre study of antifungal susceptibility patterns among 350 Candida auris isolates (2009-17) in India: role of the ERG11 and FKS1 genes in azole and echinocandin resistance. J Antimicrob Chemother. 2018;73:891-9.

34. Centers for Disease Control and Prevention. Recommendations for identification of Candida auris. [homepage na Internet]; [consultado 2019 mai 2]. Disponível em https://www.cdc.gov/fungal/candida-auris/ recommendations.html.

35. Cortegiani A, Misseri G, Fasciana T, Giammanco A, Giarratano A Chowdhary A. Epidemiology, clinical characteristics, resistance, and treatment of infections by Candida auris. J Intensive Care. 2018;6:69. eCollection 2018.

36. Arendrup MC, Prakash A, Meletiadis J, Sharma C, Chowdhary A. Comparison of EUCAST and CLSI reference microdilution MICs of eight antifungal compounds for Candida auris and associated tentative epidemiological cutoff values. Antimicrob Agents Chemother. 2017;61:e00485-17.

37. Lepak AJ, Zhao M, Berkow EL, Lockhart SR, Andes DR. Pharmacodynamic optimization for treatment of invasive Candida auris infection. Antimicrob Agents Chemother. 2017;61:e00791-17.

38. Pappas PG, Kauffman CA, Andes DR, Clancy CJ, Marr KA, OstroskyZeichner $L$, et al. Clinical practice guideline for the management of Candidiasis: 2016 update by the Infectious Diseases Society of America. Clin Infect Dis. 2016;62:e1-50.

39. Cornely OA, Bassetti M, Calandra T, Garbino J, Kullberg BJ, Lortholary O, et al. ESCMID Fungal Infection Study Group. ESCMID* guideline for the diagnosis and management of Candida diseases 2012: nonneutropenic adult patients. Clin Microbiol Infect. 2012;18:S19-37.

40. Infection Prevention and Control for Candida auris [homepage na Internet]; [consultado 2019 ago 2]. Disponível em https://www.cdc.gov/ fungal/candida-auris/c-auris-infection-control.html.

41. Candida auris: laboratory investigation, management and infection prevention and control. Guidance for the laboratory investigation, management and infection prevention and control for cases of Candida auris (C. auris) [homepage na Internet]; [consultado 2019 ago 2]; Disponível em: https://www.gov.uk/government/publications/candidaauris-laboratory-investigation-management-and-infection-preventionand-control.

42. Interim guidance for management of Candida auris infections in South African hospitals. [homepage na Internet]; [consultado 2019 ago 2]; Disponível em http://www.nicd.ac.za/assets/files/2016-12-22\%20 InterimNICDRecommdtnsCAuris.pdf.

43. Centers for Disease Control and Prevention. Global emergence of invasive infections caused by the multidrug-resistant yeast Candida auris. [homepage na Internet]; [consultado 2019 mai 8]. Disponível em https://www.cdc.gov/fungal/candida-auris/candida-auris-alert.html.

44. Snyder GM, Wright SB. The epidemiology and prevention of Candida auris. Curr Infect Dis Rep. 2019;21:19.

45. Jackson BR, Chow N, Forsberg K, Litvintseva AP, Lockhart SR, Welsh $\mathrm{R}$, et al. On the origins of a species: what might explain the rise of Candida auris? J Fungi. 2019;5:58. 